\title{
Genetic recombination is associated with intrinsic disorder in plant proteomes
}

\author{
Inmaculada Yruela ${ }^{1,2^{*}}$ and Bruno Contreras-Moreira ${ }^{1,2,3}$
}

\begin{abstract}
Background: Intrinsically disordered proteins, found in all living organisms, are essential for basic cellular functions and complement the function of ordered proteins. It has been shown that protein disorder is linked to the $G+C$ content of the genome. Furthermore, recent investigations have suggested that the evolutionary dynamics of the plant nucleus adds disordered segments to open reading frames alike, and these segments are not necessarily conserved among orthologous genes.

Results: In the present work the distribution of intrinsically disordered proteins along the chromosomes of several representative plants was analyzed. The reported results support a non-random distribution of disordered proteins along the chromosomes of Arabidopsis thaliana and Oryza sativa, two model eudicot and monocot plant species, respectively. In fact, for most chromosomes positive correlations between the frequency of disordered segments of $30+$ amino acids and both recombination rates and $G+C$ content were observed.

Conclusions: These analyses demonstrate that the presence of disordered segments among plant proteins is associated with the rates of genetic recombination of their encoding genes. Altogether, these findings suggest that high recombination rates, as well as chromosomal rearrangements, could induce disordered segments in proteins during evolution.
\end{abstract}

Keywords: Chromosome, Evolution, Intrinsically disordered proteins, Orthologues, Plant genome, Recombination rate

\section{Background}

A significant fraction of known eukaryotic genomes encode for proteins that contain regions that do not fold into a well-defined three-dimensional (3D) structure. These proteins are named intrinsically unstructured or disordered proteins (IDPs) and normally carry out signalling and regulatory functions [1-5]. These proteins might be either entirely disordered or partially disordered, characterised by regions spanning just a few $(<10)$ consecutive disordered residues (loops in otherwise well-structured proteins) or long stretches $(\geq 30)$ of contiguously disordered residues. It is thought that disordered regions confer dynamic flexibility to proteins, allowing transitions between different structural states [6]. The possible utility of such regions was first proposed by Linus Pauling [7]. More

\footnotetext{
* Correspondence: i.yruela@csic.es

${ }^{1}$ Estación Experimental de Aula Dei, Consejo Superior de Investigaciones Científicas (EEAD-CSIC), Avda. Montañana, 1005, Zaragoza 50059, Spain

${ }^{2}$ Institute of Biocomputation and Physics of Complex Systems (BIFI),

Universidad de Zaragoza, Mariano Esquillor, Edificio I+D, Zaragoza 50018, Spain

Full list of author information is available at the end of the article
}

recently, computational predictions of disordered regions have discovered that IDPs are prevalent in proteomes, and have increased during evolution. Indeed, it is predicted that $30 \%$ to $60 \%$ of proteins contain stretches of 30 or more disordered residues, being multicellular eukaryotes more enriched in predicted disordered segments than unicellular eukaryotes and prokaryotes [8]. These results suggest that proteome size, organism complexity and proteome disorder are related. Nevertheless, no overall correlations have been found apart from the clear gain in predicted disorder from prokaryotes to eukaryotes [9]. The relationship between low complexity proteins (LCPs) [10] and recombination rate has been discussed [11]. These authors suggested that the evolution of LCPs in malaria parasite Plasmodium falciparum might be related to their high genomic $\mathrm{A}+\mathrm{T}$ content and recombination rates. However, although low complexity and intrinsic disorder share some structural and sequence similarities, they are distinct phenomena [12]. There is evidence that the unstructured state, common to all living organisms, is essential for basic cellular functions linked with complex 
responses to environmental stimuli and communication between cells [9-14]. Moreover, structural disorder is critical for some protein-protein interactions, the assembly of large protein complexes and the modulation of protein activity.

The frequency of IDPs in 12 complete plant proteomes, including vascular plants, bryophyte and chlorophyta, has been previously estimated by applying the DISOPRED2 algorithm [15]. That work focused on proteins encoded by genes transferred from the chloroplast to the nucleus and reported a strong correlation between the frequency of disorder of transferred and nuclear-encoded proteins, even for polypeptides that play functional roles back in the chloroplast. Moreover, it suggested that the distribution of disordered and non-disordered segments in proteins could be to some extent random, as it showed that orthologous proteins across different species do not necessarily conserve disordered segments, despite presumably carrying out similar functions.

The evolutionary history of IDPs seems to be multiparametric, as high disorder content in proteomes has been linked to a variety of observations: $i$ ) high $\mathrm{G}+\mathrm{C}$ content, (i.e., in order to explain some exceptions in bacteria, such as Mycobacterium tuberculosis, Myxococcus xanthus and Streptomyces coelicolor) [9,16,17]; ii) expanding multi-domain protein families [9]; iii) domain arrangements [18]; or iv) alternative splicing events [19]. In order to gain additional biological and evolutionary insights into this topic, the frequency of long disordered segments among homologous proteins of 5 plant proteomes is further studied in this work. Likewise, the distribution of IDPs along the chromosomes of model eudicot (Arabidopsis thaliana) and monocot (Oryza sativa) plant species is analyzed. Finally, the possible correlations between structural disorder, genetic recombination rates and $\mathrm{G}+\mathrm{C}$ content are investigated.

\section{Results}

\section{Distribution of disordered segments along proteins}

In order to characterize the occurrence of long stretches $(\mathrm{L} \geq 30)$ of contiguously disordered residues among protein domains, their distribution in the proteomes of $A$. thaliana and $O$. sativa was investigated. Obviously, this analysis could only be performed with sequences with both annotated structural domains and predicted disordered segments, which represent $15 \%-25 \%$ of the corresponding proteomes (see Methods). The obtained results indicate that disordered segments generally (ca. 90-95\%) fall outside protein domains (Table 1). Most of these are actually in the $N$-terminal (42-54\%) and $C$-terminal (41-56\%)

Table 1 Percentages of disordered residues ${ }^{1}$ within and outside of protein domains, including linkers, $\mathbf{N}$-terminal and C-terminal regions

\begin{tabular}{|c|c|c|c|c|c|c|c|}
\hline Chromosomes & Proteins $^{2}$ & Total disorder & In domains & Outside domains & linkers $^{3}$ & $\mathrm{~N}$-term ${ }^{3}$ & C-term ${ }^{3}$ \\
\hline \multicolumn{8}{|c|}{ Arabidopsis thaliana } \\
\hline Chr1 & 1373 & $22.29 \%$ & $6.25 \%$ & $93.75 \%$ & $5.97 \%$ & $48.62 \%$ & $45.44 \%$ \\
\hline Chr2 & 770 & $21.55 \%$ & $6.46 \%$ & $93.54 \%$ & $3.92 \%$ & $54.20 \%$ & $41.87 \%$ \\
\hline Chr3 & 1023 & $22.22 \%$ & $5.81 \%$ & $94.19 \%$ & $5.16 \%$ & $47.11 \%$ & $47.85 \%$ \\
\hline Chr4 & 816 & $20.75 \%$ & $6.78 \%$ & $93.22 \%$ & $5.81 \%$ & $50.38 \%$ & $43.82 \%$ \\
\hline Chr5 & 1288 & $21.11 \%$ & $4.73 \%$ & $95.27 \%$ & $4.74 \%$ & $42.57 \%$ & $47.94 \%$ \\
\hline \multicolumn{8}{|l|}{ Oryza sativa } \\
\hline Chr1 & 792 & $21.36 \%$ & $3.43 \%$ & $96,57 \%$ & $3.43 \%$ & $47.33 \%$ & $49.24 \%$ \\
\hline Chr2 & 672 & $22.29 \%$ & $7.21 \%$ & $92.79 \%$ & $3.76 \%$ & $47.09 \%$ & $49.15 \%$ \\
\hline Chr3 & 726 & $21.21 \%$ & $6.31 \%$ & $93.69 \%$ & $4.38 \%$ & $50.07 \%$ & $45.61 \%$ \\
\hline Chr4 & 494 & $18.35 \%$ & $6.82 \%$ & $93.18 \%$ & $5.92 \%$ & $49.05 \%$ & $45.33 \%$ \\
\hline Chr5 & 465 & $22.13 \%$ & $5.84 \%$ & $94.16 \%$ & $4.95 \%$ & $45.85 \%$ & $49.43 \%$ \\
\hline Chr6 & 452 & $22.38 \%$ & $3.89 \%$ & $96.11 \%$ & $7.40 \%$ & $46.35 \%$ & $46.68 \%$ \\
\hline Chr7 & 466 & $18.97 \%$ & $5.25 \%$ & $94.75 \%$ & $6.48 \%$ & $45.49 \%$ & $48.04 \%$ \\
\hline Chr8 & 365 & $21.20 \%$ & $5.37 \%$ & $94.63 \%$ & $4.22 \%$ & $39.97 \%$ & $55.80 \%$ \\
\hline Chr9 & 309 & $20.68 \%$ & $4.36 \%$ & $95.64 \%$ & $4.75 \%$ & $41.14 \%$ & $54.19 \%$ \\
\hline Chr10 & 263 & $19.67 \%$ & $8.00 \%$ & $92.00 \%$ & $3.08 \%$ & $47.68 \%$ & $49.59 \%$ \\
\hline Chr11 & 243 & $21.31 \%$ & $4.17 \%$ & $95.83 \%$ & $4.54 \%$ & $46.22 \%$ & $49.43 \%$ \\
\hline Chr12 & 292 & $21.86 \%$ & $4.74 \%$ & $95.26 \%$ & $1.62 \%$ & $52.56 \%$ & $45.85 \%$ \\
\hline
\end{tabular}

${ }^{1}$ Included in segments of $\geq 30$ consecutive disordered amino acids over the length of the protein.

${ }^{2}$ Proteins containing Superfamily-defined domains predicted to harbour intrinsically disordered segments.

${ }^{3}$ Calculated on disordered residues outside domains. 
regions, with only (3-7\%) of disordered segments sitting in linkers that connect domains.

\section{Analysis of disorder in paralogous proteins of plants} The occurrence of protein disorder in paralogous proteins from 5 complete plant proteomes was analyzed (see Methods), including 3 eudicots and 2 monocots species. The total numbers of paralogous protein pairs analyzed were: 4566 from Arabidopsis thaliana, 7021 from Arabidopsis lyrata, 18641 from Populus trichocarpa, 4860 from Oryza sativa, and 3096 from Sorghum bicolor. The data show that on average $64 \%$ of paralogues conserve the number of predicted disordered segments, while $36 \%$ gain or lose disordered segments. No differences were observed among the plant proteomes analyzed (Table 2). When $A$. thaliana proteins were annotated with Gene Ontology (GO) terms, paralogous proteins with nonconserved disordered segments were associated with 18 biological processes and 10 molecular functions with corrected $p$-values $<10 \mathrm{E}-5$. As to the biological processes, these paralogues were mainly associated with "regulation", including regulation of nitrogen compounds (2.64E-20), nucleotide and nucleic acids (1.52E-19), RNA (3.29E-18), macromolecule biosynthesis (4.23E-17) or regulation of gene expression (6.69E-15). The most significant association among specific molecular functions was with "DNA-binding transcription factor activity" (1.71E-25), known to be frequent among IDPs $[3,5,14,20]$.

To investigate in more detail these observations a comparative study of $A$. thaliana chromosomes 1 and 4, which correspond to A. lyrata chromosomes 1 and 2, and 6 and 7 [21,22], was carried out. Particularly, homologous regions where genome rearrangements (translocations and/or inversions) presumably occurred during evolution were compared. The findings indicate that the percentage of non-conserved disordered proteins which were translocated close to the centromere is lower than that calculated for both Arabidopsis complete proteomes (33-35\%, Table 2). For instance, the results showed that $2 / 12(17 \%)$ protein coding genes sitting in the translocated region near the centromere of $A$. thaliana chromosome 4 do not conserve disordered segments. Furthermore,

Table 2 Percentages of predicted disordered segments and their conservation across plant paralogues

\begin{tabular}{lccc}
\hline Plant proteome & Paralogues & $\begin{array}{c}\text { Conserved } \\
\text { disorder }\end{array}$ & $\begin{array}{c}\text { Non-conserved } \\
\text { disorder }\end{array}$ \\
\hline A. thaliana & 4566 & $67 \%$ & $33 \%$ \\
A. lyrata & 7021 & $65 \%$ & $35 \%$ \\
P. trichocarpa & 18641 & $67 \%$ & $33 \%$ \\
O. sativa & 4860 & $62 \%$ & $38 \%$ \\
S. bicolor & 3096 & $60 \%$ & $40 \%$ \\
\hline
\end{tabular}

2/15 (13\%) proteins encoded in the S-locus do not conserve the disorder feature (Additional file 1: Figure S1). It can be reasoned that, if both the centromere and the $\mathrm{S}$-locus are reported to have low recombination rates $[21,22]$, these observations could be hinting that the frequency of disorder in proteins might be dependent on the recombination rates of their coding genes. Of course it is important to note that these regions naturally contain much reduced numbers of paralogues, therefore the estimated percentages might be inaccurate. Nevertheless, this hypothesis was tested by plotting the frequency of disordered segments of these A. thaliana and A. lyrata orthologous proteins versus the recombination rates of the corresponding chromosome fragments (Figure 1 and Additional file 1: Table S1). The obtained Pearson correlation coefficients were $r=0.693(p<0.01)$ and $r=0.881$ $(p<0.005)$, respectively, indicating that approximately half of the disorder distribution variance can be explained in terms of recombination rates. The comparison could not be extended to other chromosomes due to the lack of reported recombination rates.

Furthermore, in A. thaliana and O. sativa the results showed that disordered segments are more conserved between paralogues located in regions close to the centromere than those spanning chromosome arms (Figure 2). In particular, disordered segments were not conserved in $1 / 8$ and $2 / 10$ (ca. 12-20\%) proteins located close to the centromere of $A$, thaliana and O. sativa, respectively (A-type proteins in Figure 2). On the contrary, larger values such as $189 / 463$ and $161 / 505$ (ca. 32-40\%) or 1419/ 4056 and $1479 / 4362$ (ca. 33-36\%) were obtained for proteins located in chromosome arms (B-type and C-type proteins, respectively, in Figure 2).

\section{Genetic recombination correlates with the evolutionary} dynamics of disordered segments in A. thaliana and O. sativa The distribution of intrinsic protein disorder along the chromosomes of $A$. thaliana (5) and O. sativa (12) was first analyzed by calculating indices of dispersion. Varianceto-mean ratios $>1$ were obtained for all chromosomes, suggesting a non-random physical distribution of disordered proteins across the genome. Indeed, a structured distribution could be anticipated as protein disorder has been correlated with high genomic $\mathrm{G}+\mathrm{C}$ content in other organisms [9]. These results led us to extend the previous analyses of genomic regions within A. thaliana and A. lyrata to complete plant genomes. For that, the empirical recombination rates reported for 5 chromosomes of A. thaliana and 12 chromosomes of O. sativa (see Methods) were scatter-plotted versus the frequency of disordered segments calculated on those regions (Figure 3A and $3 \mathrm{~B})$. The average Pearson correlation coefficients calculated were $r=0.487$ for $A$. thaliana and $r=0.441$ for $O$. sativa. These statistically significant correlations 


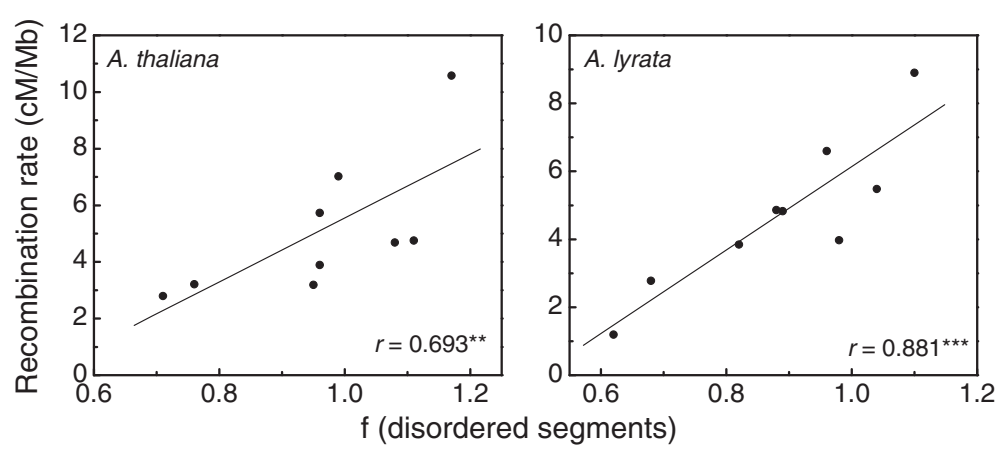

Figure 1 Scatter plot of frequency of disordered segments in proteins encoded in translocated genomic regions of $A$. thaliana and A. lyrata ( $\mathrm{X}$-axis) versus recombination rates of the corresponding chromosomic regions ( $\mathrm{Y}$-axis). A protein segment is considered disordered if it contains a contiguous stretch of predicted disordered residues of $L \geq 30$ amino acids. Statistical significance of Pearson correlation is indicated with ${ }^{* *}$ and ${ }^{* *}$, which correspond to $p<0.005$ and $p<0.01$, respectively.

show that about a quarter (19-24\%) of the overall, genomescale variance of intrinsic protein disorder distribution can be justified in terms of genetic recombination rates. As expected, this percentage is lower than that calculated for specific homologous regions of $A$. thaliana and A. lyrata (see above). However, it is noteworthy that a couple of chromosomes (chr 4 of $A$. thaliana and chr 10 of $O$. sativa) showed stronger correlations $(r=0.810$; $p<0.005$ and $r=0.772 ; p<0.005$ ), respectively). Outliers, regions where encoded proteins have a larger number of disordered segments (i.e. 5-7 segments) than their genomic contexts (i.e., 2-3 segments), were detected. At least in rice, these seem to correspond with recombination hotspots (http://rapdb.dna.affrc.go.jp/).

\section{$\mathrm{G}+\mathrm{C}$ content correlates with disordered protein segments in A. thaliana and O. sativa}

An investigation about the relationship between gene $\mathrm{G}+\mathrm{C}$ content and the frequency of disordered residues within the complete proteomes of $A$. thaliana and $O$. sativa was carried out. The $\mathrm{G}+\mathrm{C}$ content of complete nucleotide sequences $\left(G+C_{\text {total }}\right)$, disordered $\left(G+C_{\text {disordered }}\right)$ and ordered $\left(\mathrm{G}+\mathrm{C}_{\text {ordered }}\right)$ segments was calculated for all predicted IDPs (Additional file 1: Table S2). The results show that disordered regions are modestly but significantly enriched in $\mathrm{G}+\mathrm{C}$ bases $(+3.0 \%$ in $O$. sativa and $+1.6 \%$ in $A$. thaliana, $p<0.01$ ). Similar enrichments were observed in Sorghum bicolor (monocot) and Arabidopsis lyrata and Populus trichocarpa (eudicots) (Additional file 1: Table S2).

The $\mathrm{G}+\mathrm{C}_{\text {disordered }}$ frequency was plotted versus the frequency of disordered residues calculated for chromosome windows in monocot (2) and eudicot (3) plant species (Additional file 1: Figure S2). In the case of A. thaliana the obtained Pearson correlation coefficients were between $r=0.773(p<1 \mathrm{E}-4)$ for chromosome 5 and $r=0.928(p<1 \mathrm{E}-4)$ for chromosome 3 . In the case of $O$. sativa the correlation coefficients were between $r=0.737(p<1 \mathrm{E}-4)$ for chromosome 8 and $r=0.869$ $(p<1 \mathrm{E}-4)$ for chromosome 11. These results unveil a strong dependence $\left(r^{2}=0.78\right.$ for A. thaliana and $r^{2}=0.66$ for $O$. sativa) of these two variables. Similar results

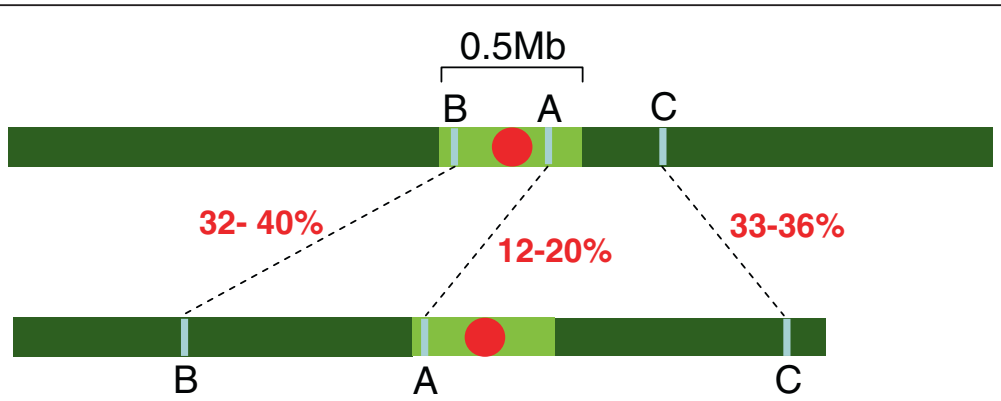

Figure 2 Diagram of two plant chromosomes with 3 types of paralogues ( $A, B$ and $C$ ) which differ in their chromosomal location. Paralogues $\mathbf{A}, \mathbf{B}$, and $\mathbf{C}$ are marked with blue bars. The percentages of non-conserved disorder between paralogues are shown. The centromere is drawn in red within a centromeric region of $0.5 \mathrm{Mb}$, shown in light green. The percentage values correspond to 1/8 and 2/10 A-type proteins, 189/463 and 161/505 B-type proteins, and 1419/4056 and 1479/4362 C-type proteins of A. thaliana and O. sativa, respectively. 
A

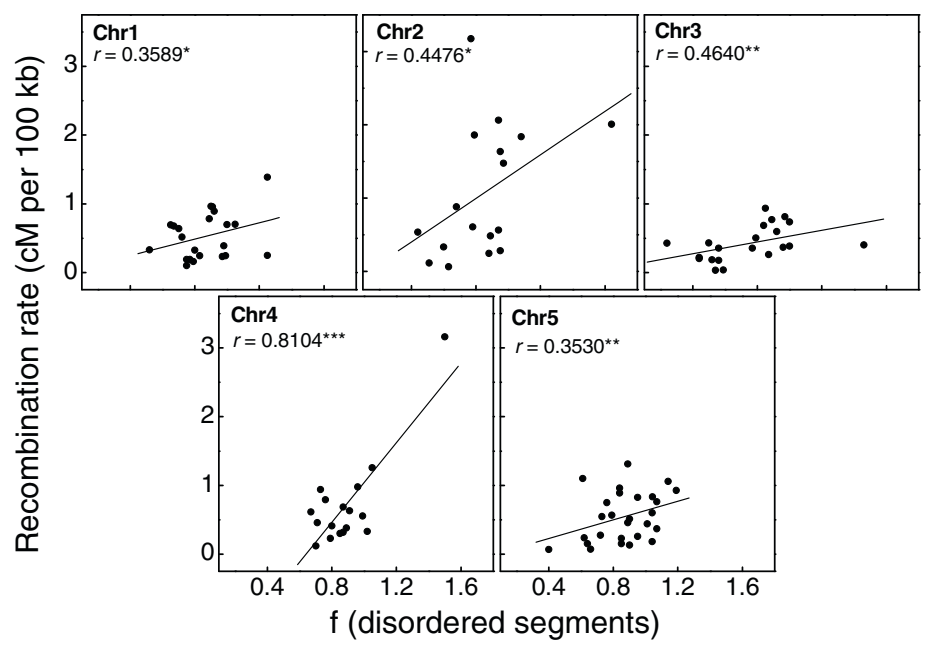

B

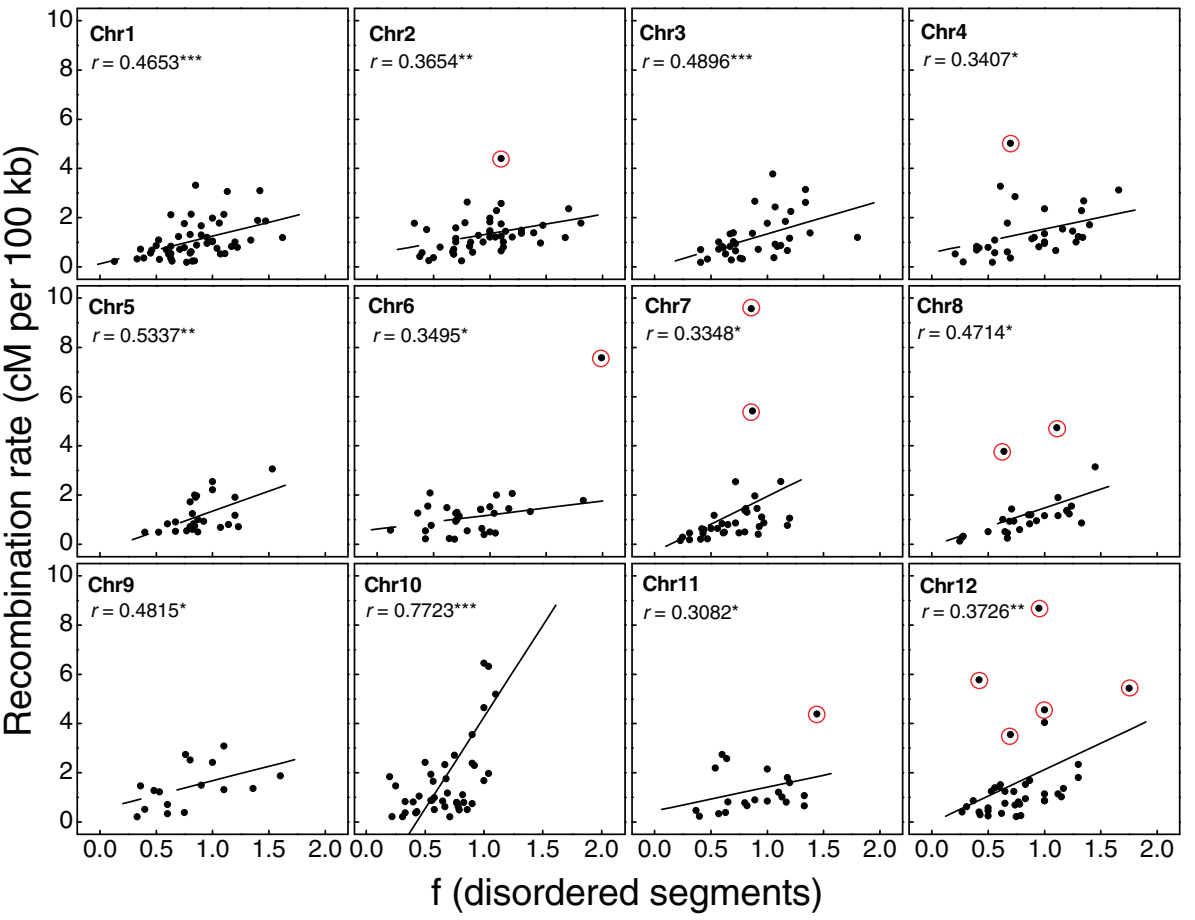

Figure 3 Scatter plot of frequency of disordered segments in the encoded proteins of mapped regions for each chromosome of A. thaliana (A) and O. sativa (B) (X-axis) and the corresponding empirical recombination rates (Y-axis). Proteins with a number of disordered segments higher than the average and associated with hotspots are marked with a red circle. Hotspots in Oryza sativa were retrieved from http://rapdb.dna.affrc.go.jp/. Statistical significance of Pearson correlation is indicated with ***, ** and *, which correspond to $p<0.005, p<$ 0.01 and $p<0.05$, respectively.

were obtained for the eudicots A. lyrata $\left(r^{2}=0.84\right)$ and P. trichocarpa $\left(r^{2}=0.76\right)$ and the monocot S. bicolor $\left(r^{2}=0.80\right)$.

\section{$\mathrm{G}+\mathrm{C}$ content correlates with recombination rates in}

\section{A. thaliana and $\mathrm{O}$. sativa}

The fact that the occurrence of protein disorder is to some extent related to recombination rates and $G+C$ content could suggest that these two later variables might also be linked. The tests carried out in this work show that they are significantly but weakly correlated, with average coefficients of $r=0.334$ in A. thaliana and $r=0.231$ in O. sativa (Figure 4A and 4B).

In order to re-assess the dependencies among protein disorder, $\mathrm{G}+\mathrm{C}$ content and recombination rates, a multiple regression analysis was performed. Taking the A. thaliana data a linear model was obtained with multiple $r^{2}=0.52$, indicating that protein disorder was only 

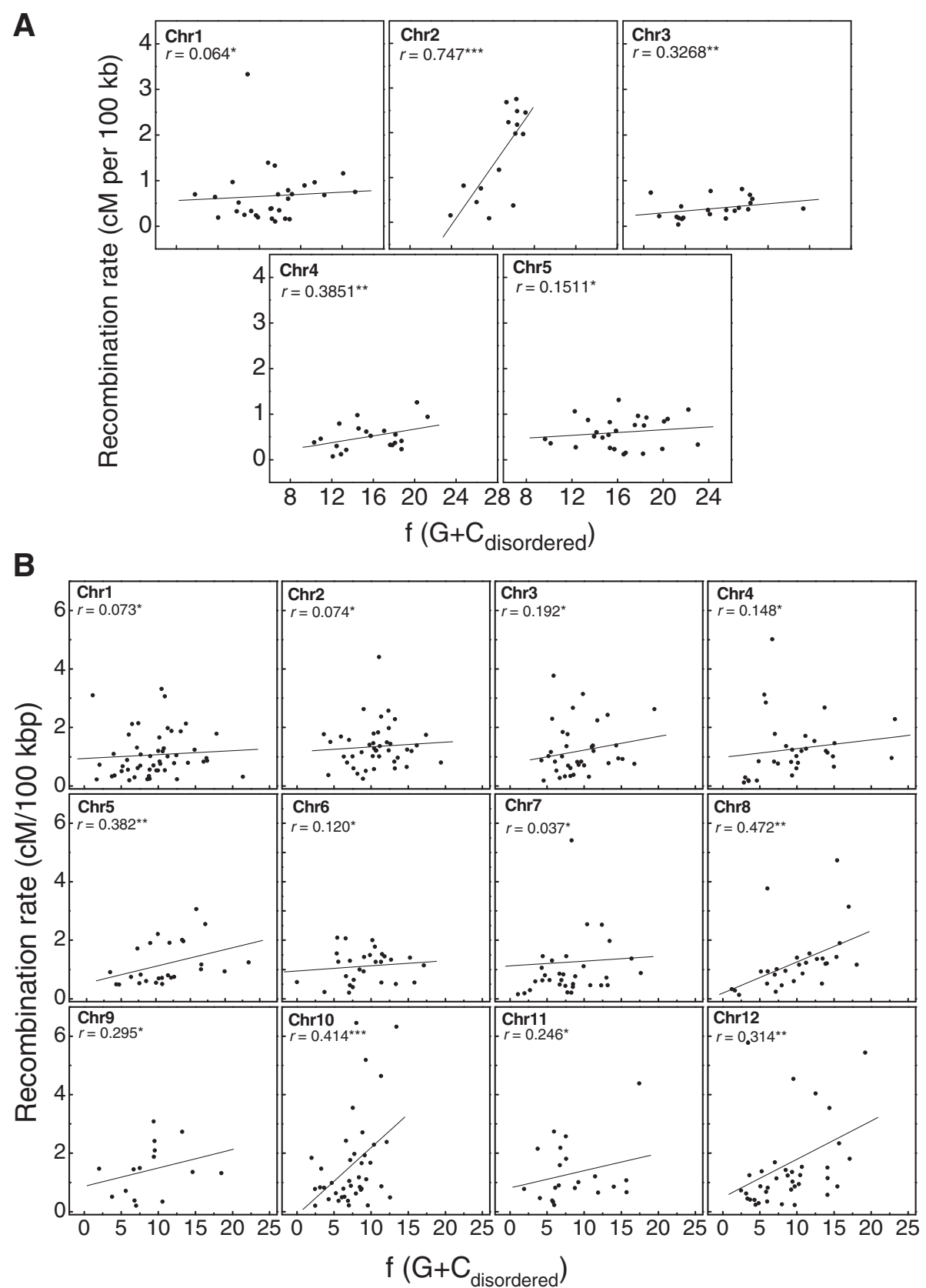

Figure 4 Scatter plots of $G+C$ disordered frequencies in genes of mapped regions for each chromosome of $A$. thaliana (A) and $O$. sativa (B) (X-axis) versus the corresponding empirical recombination rates ( $\mathrm{Y}$-axis). Statistical significance of Pearson correlation is indicated with ***, ** and *, which correspond to $p<0.005, p<0.01$ and $p<0.05$, respectively.

significantly dependent on $\mathrm{G}+\mathrm{C}$ content $(p<2 \mathrm{E}-16)$. The rice model $\left(r^{2}=0.46\right)$ confirmed the main contribution of $\mathrm{G}+\mathrm{C}$ content to protein disorder $(p<2 \mathrm{E}-16)$, but also supported a minor, but significant role of recombination rates $(p=0.013)$. Note that gene density within $A$. thaliana chromosomal regions where recombination rates have been reported is about an order of magnitude larger than in O. sativa (see Methods). These analyses required the calculation of frequencies of disordered residues as explained in Methods (Additional file 1: Figures S3A and S3B).

\section{Discussion}

In a previous paper, the analysis of 12 plant proteomes revealed a similar occurrence of IDPs to that found in other eukaryotic organisms [15], and concerning their 
taxonomic distribution, no differences were observed for IDPs among plant species. However, in some cases, homologous sequences displayed variations in the frequency of disordered segments. The inspection of 5 representative plant proteomes performed in this work indicated that on average $36 \%$ of paralogues do not conserve their composition of disordered segments. These proteins seem to be involved in regulatory processes, as most IDPs are, and therefore there is no obvious functional argument to explain their differential conservation behaviour. This result fits well with a previous study in yeast, which reported that non-conserved disordered proteins cannot be clearly associated with any function, and are expressed at low levels [5].

Gene duplication is a prominent feature in plant genome evolution with likely implications in genetic diversity and adaptation, although there is not a direct causal link between an adaptive phenotype and a specific gene duplication event because they usually occur at different times [23]. Duplicate genes arise either by regional genomic events or genome-wide polyploidization. In plants, the last is the most common mechanism. For instance, in Arabidopsis, duplications most probably resulted from a single tetraploidization event occurred some 65 million years ago [24]. This phenomenon presumably involved most genomic regions, although it has been found that centromeric regions have significantly fewer duplicated genes than chromosome arms $[25,26]$. In addition to these events, which are charted in physical maps, the available genetic maps expose the empirical recombination rates along each chromosome. It is known that recombination rates vary substantially along genomic regions. For instance, the average recombination rate ranges from $0.3 \mathrm{cM} / \mathrm{Mb}$ to $251 \mathrm{cM} / \mathrm{Mb}$ in $A$. thaliana [17] and from 0.39 to $0.42 \mathrm{cM} / \mathrm{Mb}$ in O. sativa [27]. Peak recombination rates can indicate hotspots, which are opposed to regions of suppressed recombination (coldspots). An overall positive correlation between gene density and recombination rate has been reported in model plant Brachypodium distachyon. On the contrary, a negative correlation has been observed between gene density and the frequency of repetitive regions, and rearranged chromosomal segments that retained centromeric repetitive sequences [28].

The analyses reported in this work show, for the first time, positive correlations between genetic recombination rates and protein disorder frequency in $A$. thaliana and $O$. sativa. Moreover, the results expose that certain proteins with substantially more predicted disordered segments (i.e., 5-7 segments) than the average (i.e., 2-3 segments) are located within recombination hotspots [29] (Figure 3B). These findings suggest that the physical location of paralogous genes along chromosomes could partially explain the differences found in their protein disorder composition. Genetic recombination could then be considered an evolutionary force contributing to structural disorder in proteins, at least in plants. Previous reports already discussed a relationship between low complexity proteins (LCPs) and recombination rate in Plasmodium falciparum [11]. Interestingly, in this parasite up to $50 \%$ proteins are longer than their yeast orthologues due likely to insertions or expansions of LC regions [30].

Changes in genomic architecture are a formidable force in the evolution of plants, and structural chromosome rearrangements similar to those of A. lyrata and A. thaliana $[21,22]$ are frequent. As a side-effect, these processes can drive domain sorting in proteins or the formation of novel domains [31]. Indeed, it has been reported that a significant portion of emerged novel domains during evolution are highly disordered [18]. Thus, evolutionary increase of protein disorder could be driven by modular or domain exchanges. The link between intrinsic structural disorder and modularity has been recently investigated in the human genome, finding that high levels of disorder within proteins are encoded by symmetric exons, possibly derived from internal tandem duplications [32]. The data in this work clearly indicate that disordered segments are mostly located outside annotated domains, with a similar frequency at both $N$ - and $C$ - termini, and a rather low occurrence in linker regions.

This paper reports strong positive correlations between $\mathrm{G}+\mathrm{C}$ content in coding sequences and predicted protein disorder in 5 plant proteomes. This finding is in agreement with computational studies in Archaea and Bacteria, which established relationships between $\mathrm{G}+\mathrm{C}$ composition and intrinsic protein disorder [33]. During meiotic recombination, parental chromosomes undergo either large-scale genetic exchanges by crossover or small-scale exchanges by gene conversion. There is evidence that in some eukaryote species gene conversion affecting G/C: A/T heterozygous sites yields more frequently $G / C$ than $\mathrm{A} / \mathrm{T}$ alleles. This process is known as GC-biased gene conversion (gBGC) and increases the GC content of recombining DNA over evolutionary time [34-37]. Indeed gBGC is considered the major mechanism explaining the variation of $\mathrm{G}+\mathrm{C}$ content within and between eukaryote genomes, as coding sequences rich in $\mathrm{G}+\mathrm{C}$ bases have a higher content of Arg, Gly, Ala and Pro codons, precisely those amino acids overrepresented in IDPs $[2,15,33,38]$. These composition differences explain the $\mathrm{G}+\mathrm{C}$ content reported in this work for ordered and disordered regions.

Previous papers have published strong positive correlations in human, yeast, Caenorhabditis elegans, Drosophila melanogaster and two rice species between crossover rates and $\mathrm{G}+\mathrm{C}$ composition [39-42]. On the contrary, the work of Wu et al. [27] about recombination hotspots and coldspots in O. sativa did not reveal a clear relationship 
between these two variables. Moreover, Pessia et al. [43] found no significant correlations in the genomes of A. thaliana, P. trichocarpa and Vitis vinifera and even reported a negative correlation not consistent with gBGC in S. bicolor. A negative correlation was also reported for A. thaliana chromosome 4 [44]. At first sight these apparent contradictions could be telling that the relationship between recombination and $\mathrm{G}+\mathrm{C}$ composition might be dependent on the plant species. Yet, a review of these studies reveals that $\mathrm{G}+\mathrm{C}$ measurements are not always comparable, and that recombination rates are estimated with different resolution thresholds. For instance, theoretical equilibrium $\mathrm{G}+\mathrm{C}$ values cannot be directly compared to empirical $\mathrm{G}+\mathrm{C}$ counts in sequenced genomes. Regarding this open question, this paper reports a significant but weak association between recombination and $\mathrm{G}+\mathrm{C}$ content in A. thaliana and O. sativa. When a multiple regression analysis was carried out to delineate their influence on protein disorder, clearly the effect of $\mathrm{G}+\mathrm{C}$ content was stronger than recombination. Taken together, these observations support a strong molecularbased dependency of protein disorder and $\mathrm{G}+\mathrm{C}$ content, while suggesting a much weaker relationship between $\mathrm{G}+\mathrm{C}$ and recombination. In other words, codon composition of amino acid residues common in disordered segments is directly translated into higher $G+C$ values. However, the proposed link between gBGC and $\mathrm{G}+\mathrm{C}$ content is much harder to capture with the kind of data used in this work.

\section{Conclusion}

The results demonstrate that the presence of disordered segments among plant protiens is associated with the rates of genetic recombination of their encoding genes. High recombination rates, as well as chromosomal rearrangements, could induce disordered segments in proteins during evolution. Additionally, the results indicated a stronger molecular-based dependency of protein disorder and $\mathrm{G}+\mathrm{C}$ content and much weaker dependency between $\mathrm{G}+\mathrm{C}$ content and recombination rate in plant genomes.

\section{Methods}

\section{Proteomic, GO and chromosome map databases}

Complete plant proteomes, orthology and paralogy assignments and GO annotations for Arabidopsis thaliana (AT), Oryza sativa (OS), Populus trichocarpa (PT), Sorghum bicolor (SB) were retrieved from PLAZA v.1 (http://bioinformatics.psb.ugent.be/plaza_v1/), and Arabidopsis lyrata (AL) from PLAZA v.2.5 (http://bioinformatics.psb.ugent.be/ plaza/). Note that the same data versions of a previous paper [15] were employed to facilitate comparisons to the results published here. Genetic maps from A. thaliana and $O$. sativa were retrieved from http://www.arabidopsis. org/servlets/mapper and http://rapdb.dna.affrc.go.jp/, respectively. Superfamily-defined (http://supfam.cs.bris.ac. uk) protein domains for $A$. thaliana and $O$. sativa were retrieved from http://bioinformatics.psb.ugent.be.

\section{Recombination rates}

Empirical rates of recombination in A. thaliana and O. sativa were taken from Colomé-Tatché et al. [29] and IRGSP/RAP build 5 annotation data (http://rapdb. dna.affrc.go.jp/), respectively. For Pearson correlation analyses, chromosomes were split in fragments corresponding to the regions of the genetic map where recombination rates had been empirically determined. The mean sizes of those fragments were $0.83 \mathrm{~kb} \pm 0.14$ and $0.14 \pm 0.005 \mathrm{~kb}$ for $A$. thaliana and $O$. sativa, respectively. The mean numbers of contained genes were $178 \pm 36$ and $19 \pm 7$, respectively. Recombination rates (cM) were normalized by dividing by window size $(\mathrm{Mb})$.

\section{Predictions of intrinsic disorder}

DISOPRED2 v2.42 [45] disorder predictions were performed for all protein sequences annotated in 5 plant species. All input sequences, plus the reference database uniref 90 , were low-complexity filtered with PFILT and scanned with 3 iterations of blastpgp with an E-value cut-off of 0.001 . Please check the previous paper for a benchmark on disorder predictions in plant proteins [15]. In order to put these results on a genomic scale, and to correct for regions with distinct gene density, chromosomes were split in non-overlapping windows and the observed number of disordered segments of length (L) $\geq 30$ amino acids in each window divided by the total number of open reading frames contained therein.

Frequencies of disordered residues were also computed for their direct comparison with windowed $\mathrm{G}+\mathrm{C}$ contents, which are residue-based, in a multiple regression analysis, as explained below. These frequencies were defined as the number of disordered residues contained in segments of $L \geq 30$ over the length of the encoding gene. It is worth mentioning that linear regressions using frequencies of disordered residues yield coefficients somewhat lower than those shown in Figure 3.

\section{Calculation of $\mathrm{G}+\mathrm{C}$ content}

Three variants of $G+C$ content were computed: i) $G+$ $C_{\text {total }}$, the total number of $\mathrm{G}+\mathrm{C}$ bases over the complete nucleotide sequence of a gene; ii) $G+C_{\text {disordered }}$ the number of $\mathrm{G}+\mathrm{C}$ bases spanning the predicted disordered segments in a gene; and iii) $G+C_{\text {ordered }}=G+C_{\text {total }}-G+$ $\mathrm{C}_{\text {disordered. }}$ The $\mathrm{G}+\mathrm{C}_{\text {disordered }}$ frequency was defined as $\mathrm{G}+\mathrm{C}_{\text {disordered }} /$ gene_length.

\section{Statistical analyses}

Student's $t$ tests on $\mathrm{G}+\mathrm{C}$ content were performed after checking the normality of data. The index of dispersion 
is defined as the ratio of the variance to the mean physical location of annotated genes, and was calculated for individual chromosomes. Multiple regression linear models of frequency of disordered residues as a function of both $\mathrm{G}+\mathrm{C}$ content and recombination rates were calculated with the $l m$ function of the R package (http://www.Rproject.org) with $A$. thaliana and $O$. sativa data. The obtained linear models were subsequentially evaluated with ANOVA tests in order to assess the contribution of each variable. Heteroscedasticity and normality diagnostic plots were performed to validate the model.

\section{Additional file}

Additional file 1: Figure S1. Diagram of $A$. thaliana chromosome 4 and the corresponding regions of A. lyrata chromosomes 6 and 7. The number of proteins in S-locus and translocated regions near the centromere, and the corresponding percentages of non-conserved disorder, are shown. Accession codes of proteins in S-locus region: At4g19680 (*), At4g20360, At4g20410, At4g20760, At4g20960, At4g21150 (*), At4g21340, At4g21350, At4g21430, At4g21580, At4g21800, At4g21960, At4g22200, At4g22360, At4g22720. Accession codes of proteins in the translocated region: At4g00030, At4g00660 (*), At4g02390, At4g04350, At4g05420, At4g12030, At4g10340, At4g08170 (*), At4g07390, At4g06744, At4g06599, At4g06534. Proteins marked with $\left(^{*}\right)$ do not conserve disordered segments. Table S1. Recombination rates and frequency of disordered segments in $A$. thaliana chromosomes 1 and 4, and the corresponding regions of $A$. lyrata chromosomes 1 and 2, and of A. lyrata chromosomes 6 and 7. Table S2. Percentages of $\mathrm{G}+\mathrm{C}$ content in the nucleotide sequences of encoded proteins, ordered and disordered regions. Figure S2. Scatter plot of disordered residue frequencies in proteins from each chromosome from $A$. thaliana $(A)$, A. Iyrata (B), O. sativa (C), P. trichocarpa (D) and S. bicolor (E) (X-axis) versus the $G+C_{\text {disordered }}$ frequency of their gene coding sequence $(Y$-axis). Disordered residue frequencies were calculated as the number of residues in the disordered segments of length $(L) \geq 30$ amino acids within $0.5 \mathrm{Mb}$ windows divided by the total number of residues in the open reading frames. Statistical significance of Pearson correlation is indicated with ***,** and *, which correspond to $p<0.005, p<0.01$ and $p<0.05$, respectively. Figure S3. Scatter plot of disordered residue frequency in the encoded proteins of mapped regions for each chromosome of $A$. thaliana (A) and $O$. sativa (B) (X-axis) versus the corresponding empirical recombination rates (Y-axis). Disordered residue frequencies were calculated as the number of residues in disordered segments of length $(L) \geq 30$ amino acids in each mapped chromosomic region divided by the total number of residues in the open reading frames. Statistical significance of Pearson correlation is indicated with ${ }^{* *},{ }^{* *}$ and ${ }^{*}$, which correspond to $p<0.005$, $p<0.01$ and $p<0.05$, respectively.

\section{Competing interests}

The authors declare that they have no competing interests.

\section{Authors' contributions}

IY carried out the genome analysis, participated in the design and coordination of the study, and wrote the manuscript. BC-M participated in the design of the study and the data analysis, and helped write the manuscript. Both authors have read and approved the final manuscript.

\section{Acknowledgements}

We thank S. Begueria for help in statistical analysis. This work was supported by grants from Ministerio de Economía y Competitividad (MAT2011-23861) and Gobierno de Aragón (DGA-GC B18 to I.Y. and DGA-GC A06 to B.C.-M.). All these grants were partially funded by the EU FEDER Program. We acknowledge support from CSIC Open Access Publication Support Initiative, through its Unit of Information Resources for Research (URICI), to help cover the Open Access fee.

\section{Author details}

${ }^{1}$ Estación Experimental de Aula Dei, Consejo Superior de Investigaciones Científicas (EEAD-CSIC), Avda. Montañana, 1005, Zaragoza 50059, Spain. ${ }^{2}$ Institute of Biocomputation and Physics of Complex Systems (BIFI), Universidad de Zaragoza, Mariano Esquillor, Edificio I+D, Zaragoza 50018, Spain. ${ }^{3}$ Fundación ARAID, Zaragoza, Spain.

Received: 24 May 2013 Accepted: 31 October 2013

Published: 9 November 2013

\section{References}

1. Dunker AK, Obradovic Z, Romero P, Garner EC, Brown CJ: Intrinsic protein disorder in complete genomes. Genome Inform 2000, 11:161-171.

2. Dyson HJ, Wright PE: Intrinsically unstructured proteins and their functions. Nat Rev Mol Cell Biol 2005, 6:197-208.

3. Tompa P, Taylor and Francis Group: Structure and Function of Instrinsically Disordered Proteins. Boca Raton, FL: CRC Press; 2009

4. Schlessinger A, Schaefer C, Vicedo E, Schmidberger M, Punta M, Rost B: Protein disorder - a breakthrough invention of evolution? Curr Opin Struct Biol 2011, 21:412-418.

5. Bellay J, Han S, Michaut M, Kim T, Costanzo M, Andrews BJ, Boone C, Bader GD, Myers $C L$, Kim PM: Bringing order to protein disorder through comparative genomics and genetic interactions. Genome Biol 2011, 12:R14.

6. Radivojac P, Obradovic Z, Smith DK, Zhu G, Vucetic S, Brown CJ, Lawson JD, Dunker AK: Protein flexibility and intrinsic disorder. Protein Sci 2004, 13:71-80.

7. Pauling L: A theory of the structure and process of formation of antibodies. J Am Chem Soc 1940, 62:2643-2657.

8. Radivojak P, lakoucheva LM, Oldfield CJ, Obradovic Z, Uversky VN, Dunker AK: Intrinsic disorder and functional proteomics. Biophys J 2007, 92:1439-1456.

9. Schad E, Tompa P, Hegyi H: The relationship between proteome size, structural disorder and organism complexity. Genome Biol 2011, 12:R120.

10. Wootton JC: Non-globular domains in protein sequences: automated segmentation using complexity measures. Compt Chem 1994, 17:149-163.

11. De Pristo MA, Zilversmit MM, Hartl DL: On the abundance, amino acid composition, and evolutionary dynamics of low complexity regions in proteins. Gene 2006, 378:19-30.

12. Romero P, Obradovic Z, Li X, Garner EC, Brown CJ, Dunker AK: Sequence complexity of disordered protein. Proteins 2001, 42:38-48.

13. lakoucheva LM, Brown CJ, Lawson JD, Obradovic Z, Dunker AK: Intrinsic disorder in cell-signaling and cancer-associated proteins. J Mol Biol 2002, 323:573-584.

14. Tompa P: Intrinsically unstructured proteins. Trends Biochem Sci 2002, 27:527-533.

15. Yruela I, Contreras-Moreira B: Protein disorder in plants: a view from the chloroplast. BMC Plant Biol 2012, 12:165.

16. Hegyi $H$, Tompa $P$ : Increased structural disorder of proteins encoded on human sex chromosomes. Mol Biosyst 2012, 8:229-236.

17. Singer T, Fan Y, Chang HS, Zhu T, Hazen SP, Briggs SP: A high-resolution map of Arabidopsis recombinant inbred lines by whole-genome exon array hybridization. PLOS Genet 2006, 15:e144.

18. Bornberg E, Albà MM: Dynamics and adaptive benefits of modular protein evolution. Curr Opin Struct Biol 2013. doi:10.1016/j.sbi.2013.02.012.

19. Light $\mathrm{S}$, Elofsson $\mathrm{A}$ : The impact of splicing on protein domain architecture. Curr Opin Struct Biol 2013. doi:10.1016/j.sbi.2013.02.013.

20. Dunker AK, Silman I, Uversky VN, Sussman JL: Function and structure of inherently disordered proteins. Curr Opin Struct Biol 2008, 18:756-764.

21. Kawabe A, Hansson B, Forrest A, Hagenblad J, Charlesworth D: Comparative gene mapping in Arabidopsis lyrata chromosomes 6 and 7 and A. thaliana chromosome IV: evolutionary history, rearrangements and local recombination rates. Genetical Res 2006, 88:45-56.

22. Hansson B, Kawabe A, Preuss S, Kuittinen H, Charlesworth D: Comparative gene mapping in Arabidopsis lyrata chromosomes 1 and 2 and the corresponding A. thaliana chromosome 1: recombination rates, rearrangements and centromere location. Genetical Res 2006, 87:75-85.

23. Lawton-Rauth A: Evolutionary dynamics of duplicate genes in plants. Mol Phylogenet Evol 2003, 29:396-409.

24. Ziolkowski PA, Blanc G, Sadowski J: Structural divergence of chromosomal segments that arose from successive duplication events in the Arabidopsis genome. Nucleic Acids Res 2003, 31:1339-1350. 
25. Zhang L, Gaut BS: Does recombination shape the distribution and evolution of tandemly arrayed genes (TAGs) in the Arabidopsis thaliana genome? Genome Res 2003, 13:2533-2540.

26. Kawabe A, Hansson B, Hagenblad J, Charlesworth D: Centromere locations and associated chromosome rearrangements in Arabidopsis lyrata and A. thaliana. Genetics 2006, 173(Forrest A):1613-1619.

27. Wu J, Mizuno H, Hayashi-Tsugane M, Ito Y, Chiden Y, Fujisawa M, Katagiri S, Saji S, Yoshiki S, Karasawa W, Yoshihara R, Hayashi A, Kobayashi H, Ito K, Hamada M, Okamoto M, Ikeno M, Ichikawa Y, Katayose Y, Yano M, Matsumoto T, Sasaki T: Physical maps and recombination frequency of six rice chromosomes. Plant J 2003, 36:720-730.

28. Huo N, Garvin DF, You FM, McMahon S, Luo MC, Gu YQ, Lazo GR, Vogel JP Comparison of a high-density genetic linkage map to genome features in the model grass Brachypodium distachyon. Theor Appl Genet 2011, 123:455-464.

29. Colomé-Tatché M, Cortijo S, Wardenaar R, Morgado L, Lahouze B, Sarazin A, Etcheverry M, Martin A, Feng S, Duvernois-Berthet E, Labadie K, Wincker P, Jacobsen SE, Jansen RC, Colot V, Johannes F: Features of the Arabidopsis recombination landscape resulting from the combined loss of sequence variation and DNA methylation. Proc Natl Acad Sci U S A 2012, 109:16240-16245.

30. Aravind L, lyer LM, Wellems TE, Miller LH: Plasmodium biology: genomic gleanings. Cell 2003, 115:771-785.

31. Toll-Riera M, Albà MM: Emergence of novel domains in proteins. BMC Evol Biol 2013, 13:47.

32. Schad E, Kalmar L, Tompa P: Exon-phase symmetry and intrinsic structural disorder promote modular evolution in the human genome. Nucleic Acids Res 2013, 41:4409-4422.

33. Pavlović-Lažetić GM, Mitić NS, Kovačević JJ, Obradović Z, Malkov SN, Beljanski MV: Bioinformatics analysis of disordered proteins in prokaryotes. BMC Bioinforma 2011, 12:66.

34. Eyre-Walker $A$ : Recombination and mammalian genome evolution. Proc Biol Sci 1993, 22:237-243.

35. Galtier N, Piganeau G, Mouchiroud D, Duret L: GC-content evolution in mammalian genomes: the biased gene conversion hypothesis. Genetics 2001, 159:907-911.

36. Marais $\mathrm{G}$ : Biased gene conversion: implications for genome and sex evolution. Trends Genet 2003, 19:330-338.

37. Duret L, Galtier N: Biased gene conversion and the evolution of mammalian genomic landscapes. Annu Rev Genomics Hum Genet 2003, 10:285-311

38. Uversky VN, Oldfield CJ, Dunker AK: Intrinsically disordered proteins in human diseases: introducing the $\mathrm{D}^{2}$ concept. Annu Rev Plant Physiol Plant Mol Biol 2008, 37:215-246.

39. Meunier J, Duret L: Recombination drives the evolution of GC content in the human genome. Mol Biol Evol 2004, 21:984-990

40. Muyle A, Serres-Giardi L, Ressayre A, Escobar J, Glémin S: GC-biased gene conversion and selection affect GC content in the Oryza genus (rice). Mol Biol Evol 2011, 28:2695-2706.

41. Jensen-Seaman MI, Furey TS, Payseur BA, Lu Y, Roskin KM, Chen CF, Thomas MA, Haussler D, Jacob HJ: Comparative recombination rates in the rat, mouse, and human genomes. Genome Res 2004, 14:528-538.

42. Myers S, Bottolo L, Freeman C, McVean G, Donnelly P: A fine-scale map of recombination rates and hotspots across the human genome. Science 2005 , 14:310-321.

43. Pessia E, Popa A, Mousset S, Rezvoy C, Duret L, Marais GAB: Evidence for widespread GC-biased gene conversion in eukaryotes. Genome Biol Evol 2012, 4:675-682
44. Drouaud J, Camilleri C, Bourguignon PY, Canaguier A, Bérard A, Vezon D, Giancola S, Brunel D, Colot V, Prum B, Quesneville H, Mézard C: Variation in crossing-over rates across chromosome 4 of Arabidopsis thaliana reveals the presence of meiotic recombination "hot spots". Genome Res 2006 16:106-114.

45. Ward JJ, McGuffin LJ, Bryson K, Buxton BF, Jones DT: The DISOPRED server for the prediction of protein disorder. Bioinformatics 2004, 20:2138-2139.

doi:10.1186/1471-2164-14-772

Cite this article as: Yruela and Contreras-Moreira: Genetic recombination is associated with intrinsic disorder in plant proteomes. BMC Genomics $201314: 772$

\section{Submit your next manuscript to BioMed Central and take full advantage of:}

- Convenient online submission

- Thorough peer review

- No space constraints or color figure charges

- Immediate publication on acceptance

- Inclusion in PubMed, CAS, Scopus and Google Scholar

- Research which is freely available for redistribution

Submit your manuscript at www.biomedcentral.com/submit
C Biomed Central 